Proceedings of the Edinburgh Mathematical Society (2003) 46, 329-340 (C)

DOI:10.1017/S001309150000095X Printed in the United Kingdom

\title{
UNIQUENESS IN THE CAUCHY PROBLEM FOR PARABOLIC EQUATIONS
}

\author{
ELISA FERRETTI \\ Department of Mathematics, University of Bologna, \\ Piazza di Porta San Donato 5, 40127 Bologna, Italy (ferretti@dm.unibo.it)
}

(Received 21 September 2000)

Abstract We discuss the problem of the uniqueness of the solution to the Cauchy problem for secondorder, linear, uniformly parabolic differential equations. For most uniqueness theorems the solution must be uniformly bounded with respect to the time variable $t$, but some authors have shown an interest in relaxing the growth conditions in time.

In 1997, Chung proved that, in the case of the heat equation, uniqueness holds under the restriction: $|u(x, t)| \leqslant C \exp \left[(a / t)^{\alpha}+a|x|^{2}\right]$, for some constants $C, a>0,0<\alpha<1$. The proof of Chung's theorem is based on ultradistribution theory, in particular it relies heavily on the fact that the coefficients are constants and that the solution is smooth. Therefore, his method does not work for parabolic operators with arbitrary coefficients. In this paper we prove a uniqueness theorem for uniformly parabolic equations imposing the same growth condition as Chung on the solution $u(x, t)$. At the centre of the proof are the maximum principle, Gaussian-type estimates for short cylinders and a boot-strapping argument.

Keywords: Cauchy problem; parabolic equations; uniqueness theorems; heat equation

2000 Mathematics subject classification: Primary 35K15

\section{Introduction}

In this paper we are concerned with the question of uniqueness of solutions of the initialvalue problem

$$
\begin{gathered}
L u=g \quad \text { on } \mathbb{R}^{n} \times(0, T), \\
u(x, 0)=f(x) \quad \text { on } \mathbb{R}^{n} .
\end{gathered}
$$

Here $0<T \leqslant \infty$ and $L$ is a second-order parabolic operator in divergence form (D) or non-divergence form (ND):

$$
\begin{aligned}
& L u=D_{t} u(x, t)-\sum_{i, j=1}^{n} D_{i}\left(a_{i j}(x, t) D_{j} u(x, t)\right)=u_{t}-(D, a D u), \\
& L u=D_{t} u(x, t)-\sum_{i, j=1}^{n} a_{i j}(x, t) D_{i j} u(x, t)=u_{t}-(a D, D u),
\end{aligned}
$$


where $D_{j} u=\partial u / \partial x_{j}, D_{i j} u=D_{i} D_{j} u, D_{t} u=\partial u / \partial t$ in $\mathbb{R}^{n} \times(0, T)$. We assume that the matrix of coefficients $a=a(x, t)=\left(a_{i j}(x, t)\right)$ is bounded, smooth and uniformly positive definite, that is $a_{i j}=a_{i j}(x, t) \in C^{\infty}\left(\mathbb{R}^{n} \times(0, T)\right)$ and for all $X=(x, t) \in \mathbb{R}^{n} \times(0, T)$, $\xi=\left(\xi_{1}, \ldots, \xi_{n}\right) \in \mathbb{R}^{n}$,

$$
\nu|\xi|^{2} \leqslant \sum_{i, j} a_{i j}(X) \xi_{i} \xi_{j}, \quad \max _{i, j}\left|a_{i j}(X)\right| \leqslant \nu^{-1},
$$

with a constant $\nu \in(0,1]$. The assumption $a_{i j} \in C^{\infty}$ is qualitative, in the sense that none of the estimates depend on the smoothness of $a_{i j}$. By the standard approximation technique, all the results are extended to the parabolic equations with measurable $a_{i j}$ in the divergence case, and with continuous $a_{i j}$ in the non-divergence case.

\section{Known results for the heat equation}

The initial-value problem for the heat equation consists of finding a solution $u(x, t)$ of

$$
\begin{gathered}
\left(D_{t}-\Delta\right) u(x, t)=0 \quad \text { on } \mathbb{R}^{n} \times(0, T), \\
u(x, 0)=f(x) \quad \text { on } \mathbb{R}^{n},
\end{gathered}
$$

where we require $u \in C^{2}$ for $x \in \mathbb{R}^{n}, t>0$, and $u \in C^{0}$ for $x \in \mathbb{R}^{n}, t \geqslant 0$. A formal solution is obtained immediately by Fourier transformation; in particular, if $f$ is continuous and bounded on $\mathbb{R}^{n}$, then

$$
u(x, t)=(4 \pi t)^{-n / 2} \int \exp \left(-|x-y|^{2} / 4 t\right) f(y) \mathrm{d} y .
$$

Notice that we may not have any solution at all if the initial-value function grows too fast. For example, there is a blow up in the case

$$
u(x, 0)=f(x)=\exp \left(\varepsilon|x|^{2+\varepsilon}\right), \quad \varepsilon>0 .
$$

Indeed, for arbitrarily fixed small $t$ we have the representation

$$
u(x, t)=\int f(x-y) \frac{c}{t^{n / 2}} \exp \left(-|y|^{2} / 4 t\right) \mathrm{d} y,
$$

and for $x=0$ we see that

$$
u(0, t)=c(t) \int \exp \left(\varepsilon|y|^{2+\varepsilon}-|y|^{2} / 4 t\right) \mathrm{d} y=\infty .
$$

Formula (2.3) represents only one of the infinitely many solutions of the Cauchy problem $(2.1),(2.2)$, indeed it is well known that the temperature of the infinite rod is not uniquely determined by its initial temperature, as Tychonoff's famous example shows: consider the function

$$
u(x, t)=\sum_{n=0}^{\infty} \varphi^{(n)}(t) \frac{x^{2 n}}{(2 n) !},
$$


where

$$
\varphi(t)= \begin{cases}\mathrm{e}^{-1 / t^{2}} & \text { if } t>0, \\ 0 & \text { if } t \leqslant 0 .\end{cases}
$$

It is easy to see that it satisfies the heat equation for $t>0$ and $u(x, 0+)=0$ for each $x \in \mathbb{R}$.

For the uniqueness problem, it suffices, by linearity, to consider only the homogeneous case $f \equiv 0$. With additional growth conditions there are uniqueness theorems.

\subsection{Uniform growth conditions in time}

In 1924, Holmgren [8] proved uniqueness in the class of solutions of the one-dimensional Cauchy problem satisfying

$$
|u(x, t)| \leqslant C \exp \left(a|x|^{2} \log |x|\right), \quad \text { for }|x|>1, \quad t \in \mathbb{R} .
$$

The following is the uniqueness theorem that was originally proved by Tychonoff in $1935[\mathbf{1 6}]$.

Theorem 2.1. Let $u(x, t)$ be a continuous function on $\mathbb{R}^{n} \times[0, T]$ satisfying the heat equation (2.1) and such that for some constants $a>0$ and $C>0$

$$
|u(x, t)| \leqslant C \exp \left(a|x|^{2}\right) \quad \text { in } \mathbb{R}^{n} \times[0, T] .
$$

Then $u(x, 0)=0$ implies $u(x, t) \equiv 0$ in $\mathbb{R}^{n} \times[0, T]$.

Tychonoff also constructed a non-trivial solution of the homogeneous Cauchy problem such that

$$
|u(x, t)| \leqslant C \exp \left(a|x|^{2+\varepsilon}\right), \quad \text { for }(x, t) \in \mathbb{R}^{n} \times(0, T) .
$$

In 1936, Täcklind [15] relaxed Tychonoff's condition and generalized Holmgren's, showing that there is only one solution to the Cauchy problem for the heat equation which satisfies

$$
|u(x, t)| \leqslant C \exp [a|x| p(|x|)] \quad \text { for } t \in(0, T), \quad|x|>1,
$$

where the function $p(r)$ is any positive continuous function on $\mathbb{R}^{+}$such that

$$
\int^{\infty} \frac{\mathrm{d} r}{\inf _{s \geqslant r} p(s)}=+\infty
$$

It is easy to see that choosing $p(r)=r \log r$ we obtain (HC), while choosing $p(r)=$ $r$ we obtain (TyC). Täcklind demonstrates necessity of condition ( $\mathrm{TaC})$ by explicitly constructing a non-trivial solution of the homogeneous problem using Carleman's theory of analytic functions.

It is remarkable that even a unilateral condition, a lower bound for $u$, can ensure uniqueness. The basic result here is due to Widder [17], and states that there is at most one solution $u$ which is non-negative (for example, when $u$ is the absolute temperature) in the infinite strip $\mathbb{R} \times[0, T]$. 


\subsection{Non-uniform growth conditions in time}

For most uniqueness theorems the solution $u(x, t)$ must be uniformly bounded with respect to the $t$ variable. Some authors have shown an interest in relaxing the growth conditions in time.

In 1966, Shapiro [14] showed that if the solution $u(x, t)$ of the heat equation is bounded in every substrip of the form $0<t_{0}<t<T$ and if $\|u(x, t)\|_{\infty}=o\left(t^{-1}\right)$ for $t \rightarrow 0^{+}$, then $u \equiv 0$. In 1994, Chung and Kim [3] needed to strengthen the continuity assumption on the solution, replacing it with the hypothesis

$$
\lim _{t \rightarrow 0^{+}} \int u(x, t) \phi(x) \mathrm{d} x=0,
$$

for all $\phi \in C^{\infty}\left(\mathbb{R}^{n}\right)$ such that for some fixed $a>0$ and for every $h>0$,

$$
\sup _{x \in \mathbb{R}^{n}, \alpha} \frac{\left|\partial^{\alpha} \phi(x)\right| \exp (2 a|x|)}{h^{|\alpha|} \alpha !}<\infty
$$

to be able to show that there is only one solution satisfying (CK1) and, for $C>0$,

$$
|u(x, t)| \leqslant C \exp \left[a\left(\frac{1}{t}+|x|^{2}\right)\right] \quad \text { in } \mathbb{R}^{n} \times(0, T) .
$$

In 1999, Chung [2] went back to the classical continuity hypothesis and proved the following theorem.

Theorem 2.2. Let $u(x, t)$ be a continuous function on $\mathbb{R}^{n} \times[0, T]$ satisfying the heat equation (2.1) in $\mathbb{R}^{n} \times(0, T)$ and the following conditions.

(i) There exist constants $a>0,0<\alpha<1$ and $C>0$ such that

$$
|u(x, t)| \leqslant C \exp \left[\left(\frac{a}{t}\right)^{\alpha}+a|x|^{2}\right] \quad \text { in } \mathbb{R}^{n} \times(0, T) .
$$

(ii) $u(x, 0)=0$ for $x \in \mathbb{R}^{n}$.

Then $u(x, t) \equiv 0$ in $\mathbb{R}^{n} \times[0, T]$.

The proof of this theorem is based on ultradistribution theory, in particular it relies heavily on the fact that the coefficients are constants and the solution is smooth. Therefore, it is not possible to generalize such a proof to parabolic operators with non-constant coefficients via standard comparison techniques.

The growth condition on the space variable is optimal since it is known (see [16] or [6]) that for every $\varepsilon>0$ there exists a $C^{\infty}$ function $u(x, t)$ not identically zero, satisfying

(a) $\left(\Delta-D_{t}\right) u(x, t)=0$ on $\mathbb{R}^{n} \times(0, T)$;

(b) $u$ is continuous on $\mathbb{R}^{n} \times[0, T)$;

(c) $|u(x, t)| \leqslant C_{\varepsilon} \exp \left(|x|^{2+\varepsilon}\right)$ on $\mathbb{R}^{n} \times(0, T)$; and

(d) $u(x, 0)=0$ on $\mathbb{R}^{n}$. 
Chung and Kim [3] also prove that the growth condition in the time variable is the optimal one by showing that Theorem 2.2 fails if we replace the assumption $0<\alpha<1$ with $\alpha=1$.

The example they have obtained is the following.

\subsection{Example}

Denote by $\Gamma(x, t)$ the fundamental solution of the heat equation:

$$
\Gamma(x, t)= \begin{cases}(4 \pi t)^{-1 / 2} \exp \left(-|x|^{2} / 4 t\right) & \text { for } t>0 \\ 0 & \text { for } t \leqslant 0 .\end{cases}
$$

Let $D_{K}$ be a domain in the complex plane $\mathbb{C}$ given by

$$
D_{K}=\{z \in \mathbb{C} \mid z=x+\mathrm{i} y, x \geqslant K,-\pi \leqslant y \leqslant \pi\}, \quad K \geqslant 0,
$$

and let $C_{K}$ be the boundary of $D_{K}$. Define a function $u(x, t)$ on $\mathbb{R} \times(0, \infty)$ by

$$
u(x, t)=\frac{1}{2 \pi \mathrm{i}} \int_{C_{K}} \Gamma(x-\zeta, t) \exp \left(\mathrm{e}^{\zeta}\right) \mathrm{d} \zeta,
$$

where the integral is taken counterclockwise. Such an integral is convergent because the function $\exp \left(\mathrm{e}^{\zeta}\right)$ decreases very rapidly as $\operatorname{Re} \zeta \rightarrow \infty$ along the curve $C_{K}$ and it is easy to see that $u(x, t)$ satisfies the heat equation in $\mathbb{R} \times(0, \infty)$. Moreover, by Cauchy's Integral Theorem, $u(x, t)$ does not depend on $K \geqslant 0$. We have

$$
|u(x, t)| \leqslant A(K) \sup _{\zeta \in D_{K}}|\Gamma(x-\zeta, t)|,
$$

where

$$
A(K)=\frac{1}{2 \pi} \int_{C_{K}}\left|\exp \left(\mathrm{e}^{\zeta}\right)\right||\mathrm{d} \zeta|<\infty
$$

If we write $\zeta=\xi+\mathrm{i} \eta$, the condition $\zeta \in D_{K}$ is equivalent to $\xi \geqslant K$ and $|\eta| \leqslant \pi$, therefore we have

$$
\begin{aligned}
\sup _{\zeta \in D_{K}}|\Gamma(x-\zeta, t)| & =\frac{1}{\sqrt{4 \pi t}} \sup _{\zeta \in D_{K}} \exp \left[-\frac{(x-\xi)^{2}-\eta^{2}}{4 t}\right] \\
& =\frac{1}{\sqrt{4 \pi t}} \exp \left(\frac{\pi^{2}}{4 t}\right) \sup _{\xi \geqslant K} \exp \left[-\frac{(x-\xi)^{2}}{4 t}\right] .
\end{aligned}
$$

Then there is a constant $a>0$ such that

$$
|u(x, t)| \leqslant A(K) \exp \left(\frac{a}{t}\right) \exp \left[-\frac{d\left(x, D_{K}\right)^{2}}{4 t}\right]
$$

where $d$ is the Euclidean distance.

It follows that

$$
|u(x, t)| \leqslant A(K) \exp \left(\frac{a}{t}\right) \quad \text { in } \mathbb{R} \times(0, \infty) .
$$


Now let $r>0$ be arbitrarily fixed and $x \leqslant r$. Since the integral (2.4) is independent of $K$ we may choose $K>0$ large enough so that $(K-r)^{2}>4 a$.

Then (2.5) implies

$$
\sup _{x \leqslant r}|u(x, t)| \leqslant A(K) \exp \left[\frac{4 a-(K-r)^{2}}{4 t}\right], \quad \text { for } t>0 .
$$

This shows that $u(x, t)$ converges uniformly to 0 as $t$ approaches $0^{+}$in every half-line $(-\infty, r]$, because the right-hand side of $(2.7)$ converges to 0 as $t \rightarrow 0^{+}$. Therefore, we obtain the continuity of $u(x, t)$ in $\mathbb{R} \times[0, \infty)$, and $u(x, 0)=0$.

It remains to show that $u(x, t)$ is not identically zero.

Suppose that $u(x, t) \equiv 0$ in $\mathbb{R} \times[0, \infty)$. Then from (2.4) it follows that

$$
\int_{C_{K}} \exp \left[-\frac{(x-\zeta)^{2}}{4 t}\right] \exp \left(\mathrm{e}^{\zeta}\right) \mathrm{d} \zeta \equiv 0
$$

in $\mathbb{R} \times[0, \infty)$. Applying the Lebesgue-dominated convergence theorem, because, for fixed $x, \exp \left[-(x-\zeta)^{2} / 4 t\right]$ increases to 1 as $t \rightarrow \infty$, we can see that

$$
\int_{C_{K}} \exp \left(\mathrm{e}^{\zeta}\right) \mathrm{d} \zeta=0
$$

Because the integral expression in (2.4) does not depend on $K \geqslant 0$, we may choose $K=0$. Then $(2.8)$ can be written as

$$
\begin{aligned}
0 & =-\int_{0}^{\infty} \exp \left(-\mathrm{e}^{\xi}\right) \mathrm{d} \xi-\mathrm{i} \int_{-\pi}^{\pi} \exp \left(\mathrm{e}^{\mathrm{i} \eta}\right) \mathrm{d} \eta+\int_{0}^{\infty} \exp \left(-\mathrm{e}^{\xi}\right) \mathrm{d} \xi \\
& =-2 \mathrm{i} \int_{0}^{\pi} \exp (\cos \eta) \cos (\sin \eta) \mathrm{d} \eta
\end{aligned}
$$

and this leads to a contradiction, since $\exp (\cos \eta) \cos (\sin \eta)>0$ on $[0, \pi]$.

Therefore, $u(x, t)$ cannot be identically zero.

\section{Uniqueness theorems for parabolic equations}

For more general parabolic operators with bounded coefficients several uniqueness results are known, but all of them control the growth of the solution uniformly in time. We mention only a few.

\subsection{Uniform growth conditions in time}

In 1941, Krzyański [9] showed that the Tychonoff condition (TyC) guarantees uniqueness for parabolic operators in non-divergence form provided the coefficients are bounded 
and continuous. The extension of Widder's uniqueness theorem to solutions of equations of the form $u_{t}=a(x) u_{x x}+b(x) u_{x}+c(x) u$ with Hölder continuous and uniformly bounded coefficients is due to Serrin [13]. In 1958, working with systems of parabolic equations with bounded and sufficiently regular coefficients (e.g. $C^{2, \alpha}$ ), Zolotarev [18] used Täcklind techniques to improve Krzyański's result and demonstrate that there is uniqueness if ( $\mathrm{TaC}$ ) holds. The regularity of the coefficients was needed to assure the existence of a Green function from which some crucial estimates were derived. Under the same regularity assumptions (to guarantee existence of the Green function) on the coefficients of uniformly parabolic equations, Friedman (see [6] or [5]) proved uniqueness in the class of solutions satisfying the following integral condition:

$$
\int_{0}^{T} \int_{\mathbb{R}^{n}} \exp \left(-c|x|^{2}\right)|u(x, t)| \mathrm{d} x \mathrm{~d} t<\infty, \quad \text { where } c>0 .
$$

Also, in 1966, Aronson and Besala [1] imposed an integral condition to prove uniqueness of the solution for equations in divergence form, under mild assumptions on the coefficients:

$$
\int_{0}^{T} \int_{\mathbb{R}^{n}} \exp \left(-c|x|^{2}\right) u^{2}(x, t) \mathrm{d} x \mathrm{~d} t<\infty, \quad \text { with } c>0 .
$$

In 1978, Hayne [7], studying the non divergence case, was able to remove Zolotarev's regularity assumption showing that uniqueness holds in the Täcklind class (TaC) only when restrictions are imposed on the magnitude of the coefficients. He could derive crucial estimates constructing a comparison function independent of coefficient regularity through Carlo Pucci's theory of elliptic extremal operators. In 1995, Polidoro [11] proved that there is a unique non-negative or Tychonoff's class solution to ultraparabolic equations in divergence form of type $L u=u_{t}-(D, a D u)-(x, b D u)$, where $a=a^{\mathrm{T}}$ and $b$ is a constant matrix. He relied on pointwise estimates for the fundamental solution of the operator $L$.

Now we want to show that integral conditions of type (FC) or (AC) are equivalent to the pointwise condition $(\mathrm{TyC})$. For this purpose we will need the following Moser-type estimates for non-negative solutions of equations in (D) or (ND) form (see [10] for (D), see $[4]$ for the general case).

Theorem 3.1. Let $u$ be a non-negative solution of $L u \leqslant 0$ on a standard cylinder $C_{2 \rho}$, for some $\rho>0$. Then for each $p>0$

$$
\sup _{C_{\rho}} u \leqslant N\|u\|_{\mathcal{L}^{p}\left(C_{2 \rho}\right)}
$$

where $N=N(n, \nu, p)>0$.

Theorem 3.2. Let $u$ be a solution to $L u=0$ in $\mathbb{R}^{n} \times(0, T)$. Then, for a suitable choice of constants $b, c, B, C>0$ and for any $p>0$ we have

$$
\begin{gathered}
\int_{0}^{T} \int_{\mathbb{R}^{n}} \exp \left(-c|x|^{2}\right)|u(x, t)|^{p} \mathrm{~d} x \mathrm{~d} t<C<\infty \quad \Longleftrightarrow \\
|u(x, t)| \leqslant B \exp \left(b|x|^{2}\right) \quad \text { in } \mathbb{R}^{n} \times(0, T) .
\end{gathered}
$$


Proof. For simplicity we take $p=1$.

$(\Rightarrow)$. Suppose first that $u \geqslant 0$ in $\mathbb{R}^{n} \times(0, T)$. Let $X_{0}=\left(x_{0}, t_{0}\right)$ be arbitrarily fixed in $\mathbb{R}^{n} \times(0, T)$, with $\left|x_{0}\right|=r$. Then $C_{\rho}\left(X_{0}\right)=B_{\rho}\left(x_{0}\right) \times\left(t_{0}-\rho^{2}, t_{0}\right) \subset \mathbb{R}^{n} \times(0, T)$ for some $\rho>0$ and after rescaling we can assume $\rho=1$. By the estimate (3.1), there is $N=N(n, \nu)>0$ such that

$$
\begin{aligned}
u\left(X_{0}\right) & \leqslant \sup _{C_{1 / 2}} u \leqslant N\|u\|_{\mathcal{L}^{1}\left(C_{1}\right)}^{1}=N \iint_{C_{1}}|u| \mathrm{d} x \mathrm{~d} t \\
& \leqslant N \iint_{C_{1}}|u| \frac{\mathrm{e}^{-|x|^{2}}}{\min _{C_{1}} \mathrm{e}^{-|x|^{2}}} \mathrm{~d} x \mathrm{~d} t \\
& \leqslant N \mathrm{e}^{(r-1)^{2}} \iint_{C_{1}}|u| \mathrm{e}^{-|x|^{2}} \mathrm{~d} x \mathrm{~d} t \\
& \leqslant N C \mathrm{e}^{(r-1)^{2}} \leqslant B \mathrm{e}^{b\left|x_{0}\right|^{2}},
\end{aligned}
$$

for suitably chosen $B$ and $b$. Since $X_{0}$ is any point in the infinite strip we are done in the case $u \geqslant 0$.

If $u$ has arbitrary sign, then we set $v=u^{2}$ to get

$$
L v=L\left(u^{2}\right)=2 u L u-2(D u, a D u) \leqslant-2 \nu|D u|^{2} \leqslant 0,
$$

so that $v$ satisfies the hypothesis of Theorem 3.1. Then, proving the above estimate for $v$ is equivalent to proving it for $u$.

$(\Leftarrow)$. It is easy to see that for any $c>b$ and some $C>0$ we have

$$
\int_{0}^{T} \int_{\mathbb{R}^{n}} \exp \left(-c|x|^{2}\right)|u(x, t)| \mathrm{d} x \mathrm{~d} t \leqslant B \iint \exp \left[(b-c)|x|^{2}\right] \mathrm{d} x \mathrm{~d} t \leqslant C .
$$

\subsection{Alternative proofs of classical results}

Here we give an alternative proof of two theorems that take care of the uniqueness problem in the class of solutions satisfying $|u(x, t)| \leqslant N \mathrm{e}^{N|x|^{2}}$ or the unilateral condition $u(x, t) \geqslant-N \mathrm{e}^{-N|x|^{2}}$. The proofs are based mainly on the following known estimate for short cylinders (see [12, Theorem 2.1]).

Theorem 3.3 (estimates for short cylinders). Let $Q=B_{r}(0) \times(0, h)$ be a cylinder with $h \ll r^{2}$ and $u$ be a solution of $L u=0$ in $Q, u=0$ on $\partial_{t} Q=B_{r}(0) \times\{0\}$. Then, for any $t \in[0, h]$ we have

$$
|u(0, t)| \leqslant \exp \left(-\beta \frac{r^{2}}{h}\right) \sup _{\partial_{x} Q}|u|
$$

where $\beta=\beta(n, \nu)>0$. 
The first theorem gives uniqueness of the solution in the Tychonoff class (TyC) for the homogeneous Cauchy problem in both divergence and non-divergence cases.

Theorem 3.4. Let $u$ be a solution of the Cauchy problem

$$
\begin{aligned}
L u(x, t)=0 & & \text { in } \mathbb{R}^{n} \times(0, T), \\
u(x, 0)=0 & & \text { on } \mathbb{R}^{n},
\end{aligned}
$$

where $0<T \leqslant \infty$ and $L$ is a uniformly parabolic operator of the form $(D)$ or $(N D)$. In addition, assume

$$
|u(x, t)| \leqslant N \mathrm{e}^{N|x|^{2}} \quad \text { in } \mathbb{R}^{n} \times(0, T) .
$$

Then $u \equiv 0$.

Proof. Let $0<h \ll 1$ be fixed and take any $X=(x, t) \in \mathbb{R}^{n} \times(0, h)$. For $r \geqslant 2|x|$ consider the cylinder $B_{r}(x) \times(0, h)$ and apply Theorem 3.3 to get

$$
\begin{aligned}
|u(x, t)| & \leqslant \exp \left(-\beta \frac{r^{2}}{h}\right) \sup _{\partial B_{r}(x) \times[0, h]}|u| \\
& \leqslant \exp \left(-\beta \frac{r^{2}}{h}\right) \sup _{\partial B_{2 r}(0) \times[0, h]}|u|,
\end{aligned}
$$

where the last inequality comes from the fact that $B_{r}(x) \subset B_{2 r}(0)$ and by using the Maximum Principle. Now by (3.8), (3.9) and by assumption (3.7) we have

$$
\begin{aligned}
|u(x, t)| & \leqslant N \exp \left(N 4 r^{2}-\beta r^{2} / h\right) \\
& =N \exp \left[r^{2}(4 N-\beta / h)\right] \underset{r \rightarrow \infty}{\longrightarrow} 0
\end{aligned}
$$

when we take $0<h=h(n, \nu)<(\beta / 4 N)$. Then $u(X)=0$ for each $X \in \mathbb{R}^{n} \times(0, h)$, that is $u \equiv 0$ in $\mathbb{R}^{n} \times[0, h]$.

Repeating the same argument in the strip $\mathbb{R}^{n} \times[h, 2 h]$ we get $u \equiv 0$ on $\mathbb{R}^{n} \times[0,2 h]$, then on $\mathbb{R}^{n} \times[0,3 h]$, and so on until we obtain $u \equiv 0$ in $\mathbb{R}^{n} \times[0, T]$.

\subsection{Non-uniform growth conditions in time}

We will present a uniqueness theorem for uniformly parabolic equations imposing on the solution $u(x, t)$ the same growth condition as Chung for the heat equation (ChC). At the centre of the proof are again the estimates for short cylinders (3.4) of Theorem 3.3.

Theorem 3.5. Let $u(x, t)$ be a solution of the uniformly parabolic equation $L u=0$ on $\mathbb{R}^{n} \times(0, T)$, where $L$ is in the form $(D)$ or $(N D)$, such that $u(x, t)$ is a continuous function on $\mathbb{R}^{n} \times[0, T], u(x, 0) \equiv 0$ and for some constants $a>0,0<\alpha<1$, we have

$$
|u(x, t)| \leqslant \exp \left[a\left(t^{-\alpha}+|x|^{2}\right)\right] \quad \text { on } \mathbb{R}^{n} \times(0, T] .
$$

Then $u(x, t) \equiv 0$ in $\mathbb{R}^{n} \times[0, T]$. 
Proof. We follow a 'backward-induction' argument.

For arbitrary $R_{0} \gg 1$ and $j=0,1,2, \ldots$, we set

$$
h_{j}=4^{-j} h_{0}, \quad r_{j}=2^{(\alpha-1) j} R_{0}, \quad R_{j}=R_{0}+r_{1}+r_{2}+\cdots+r_{j},
$$

where $h_{0}$ is a sufficiently small constant. Since $0<\alpha<1$, for each $j$ we have

$$
R_{j} \leqslant R_{0}+\sum_{j=0}^{\infty} r_{j} \leqslant N_{0} R_{0}
$$

for $N_{0}=2 /\left(2-2^{\alpha}\right)>0$, and notice that

$$
\frac{r_{j}^{2}}{h_{j-1}}=\frac{4^{(\alpha-1) j} R_{0}^{2}}{4^{1-j} h_{0}}=4^{\alpha j-1} \frac{R_{0}^{2}}{h_{0}} .
$$

By assumption $u(x, t) \rightarrow 0$, as $t \rightarrow 0^{+}$, uniformly on compact subsets of $\mathbb{R}^{n}$, therefore we can fix $J \gg 1$ such that $|u(x, t)| \leqslant 1$ in $B_{N_{0} R_{0}}(0) \times\left[0, h_{J}\right]$. In particular we have

$$
|u(x, t)| \leqslant 1 \text { in } B_{R_{J}}(0) \times\left[0, h_{J}\right] .
$$

Obviously, if $t \in\left[h_{J}, h_{0}\right]$, then $t^{-\alpha}<h_{J}^{-\alpha}$, so that from $\left(B_{J}\right)$ and our growth condition (3.10) we immediately obtain

$$
|u(x, t)| \leqslant \exp \left[a\left(h_{J}^{-\alpha}+N_{0}^{2} R_{0}^{2}\right)\right] \quad \text { in } B_{R_{J}}(0) \times\left[0, h_{0}\right] .
$$

Next we want to show that $\left(A_{J}\right) \Rightarrow\left(B_{J-1}\right)$.

From the Maximum Principle and because $u \equiv 0$ on $\mathbb{R}^{n} \times\{0\}$, to estimate $u(x, t)$ in $B_{R_{J-1}}(0) \times\left[0, h_{J-1}\right]$ it suffices to take $|x|=R_{J-1}$.

Considering the cylinder of axis $|x|=R_{J-1}$, height $h_{J-1}$ and radius $r_{J}$, and applying the Gaussian estimates (3.4) in Theorem 3.3, with

$$
h=h_{J-1}=4^{1-J} h_{0} \ll r_{J}^{2}=4^{\alpha J-J},
$$

we have, for $|x|=R_{J-1}, 0<t \leqslant h_{J-1}$,

$$
\begin{aligned}
|u(x, t)| & \leqslant \exp \left[a\left(h_{J}^{-\alpha}+N_{0}^{2} R_{0}^{2}\right)\right] \exp \left(-\frac{\beta r_{J}^{2}}{h_{J-1}}\right) \\
& \leqslant \exp \left[a\left(4^{\alpha J} h_{0}^{-\alpha}+N_{0}^{2} R_{0}^{2}\right)-\beta 4^{\alpha J-1} h_{0}^{-1} R_{0}^{2}\right] \\
& \leqslant \exp \left[R_{0}^{2}\left(-\beta 4^{\alpha J-1} h_{0}^{-1}+a N_{0}^{2}\right)+a 4^{\alpha J} h_{0}^{-\alpha}\right] \\
& \leqslant 1 .
\end{aligned}
$$

The last inequality occurs because the exponent has a negative sign, provided $0<h_{0}<$ $\min \left\{1,\left(\beta / 4 a N_{0}^{2}\right)\right\}$ and $R_{0}$ is large enough.

Thus from $\left(A_{J}\right)$ it follows that

$$
|u(x, t)| \leqslant 1, \quad \text { in } B_{R_{J-1}}(0) \times\left[0, h_{J-1}\right] . \quad \quad\left(B_{J-1}\right)
$$


Therefore,

$$
\left(B_{J}\right) \Rightarrow\left(A_{J}\right) \Rightarrow\left(B_{J-1}\right) \Rightarrow \cdots \Rightarrow\left(A_{1}\right) \Rightarrow\left(B_{0}\right)
$$

where

$$
|u(x, t)| \leqslant 1, \quad \text { for }|x| \leqslant R_{0}, \quad 0 \leqslant t \leqslant h_{0} .
$$

Since $R_{0}$ is arbitrarily large, taking the limit for $R_{0} \rightarrow \infty$, we obtain $|u(x, t)| \leqslant 1$ on $\mathbb{R}^{n} \times\left[0, h_{0}\right]$.

Then by Theorem 3.4 we have $u \equiv 0$ in $\mathbb{R}^{n} \times\left[0, h_{0}\right]$. Repeating the argument in the strip $\mathbb{R}^{n} \times\left[h_{0}, 2 h_{0}\right]$, then on $\mathbb{R}^{n} \times\left[2 h_{0}, 3 h_{0}\right]$ and so on, we will finally get $u \equiv 0$ on $\mathbb{R}^{n} \times[0, T]$.

Acknowledgements. The author was partly supported by NSF grant no. DMS9623287.

\section{References}

1. D. G. Aronson and P. Besala, Uniqueness of solutions of the Cauchy problem for parabolic equations, J. Math. Analysis Applic. 13 (1966), 516-526.

2. S.-Y. Chung, Uniqueness in the Cauchy problem for the heat equation, Proc. Edinb. Math. Soc. 42 (1999), 455-468.

3. S.-Y. Chung And D. Kim, An example of non uniqueness of the Cauchy problem for the heat equation, Commun. PDEs 19 (1994), 1257-1261.

4. E. FERREtTi AND M. V. SAFOnov, Growth theorems and Harnack inequality for second order parabolic equations, Contemp. Math. 277 (2001), 87-112.

5. A. Friedman, On the uniqueness of the Cauchy problem for parabolic equations, Am. J. Math. 81 (1959), 503-511.

6. A. Friedman, Partial differential equations of parabolic type (Prentice Hall, 1964).

7. R. M. HaYne, Uniqueness in the Cauchy problem for parabolic equations, Trans. Am. Math. Soc. 241 (1978), 373-399.

8. E. Holmgren, Sur le solutions quasianalytiques de l'équation de la chaleur, Ark. Mat. 138 (1924), 1-9.

9. M. KRZYAŃSKI, Sur les solutions des equations du type parabolique determinées dans une région illimitée, Bull. Am. Math. Soc. 47 (1941), 911-915.

10. J. Moser, A Harnack inequality for parabolic differential equations, Commun. Pure Appl. Math. 17 (1964), 101-134 (and correction in Commun. Pure Appl. Math. 20 (1967), 231236).

11. S. Polidoro, Uniqueness and representation theorems for solutions of KolmogorovFokker-Planck equations, Rend. Mat. VII 15 (1995), 535-560.

12. M. V. SAFONOV, Behavior near the boundary of positive solutions of second order parabolic equations, Documenta Matematica, Extra Volume ICM 1998, vol. 1, pp. 637-647.

13. J. B. SERRIN, A uniqueness theorem for the parabolic equation $u_{t}=a(x) u_{x x}+b(x) u_{x}+$ $c(x) u$, Bull. Am. Math. Soc. 60 (1954), 344.

14. V. L. Shapiro, The uniqueness of solutions of the heat equation in an infinite strip, Trans. Am. Math. Soc. 125 (1966), 326-335.

15. S. TäCKLIND, Sur les classes quasianalytiques des solutions des équationsaux derivées partielles du type parabolique, Nova Acta Soc. Sci. Upsal. 10 (1936), 1-57.

16. A. N. Tychonoff, A uniqueness theorem for the heat equation, Mat. Sb. 42 (1935), 199-216. 
17. D. V. Widder, Positive temperatures on the infinite rod, Trans. Am. Math. Soc. $\mathbf{5 5}$ (1944), 85-95.

18. G. N. Zolotarev, The uniqueness of the solution of the Cauchy problem for systems parabolic in the sense of I. G. Petrowski, Izv. Vyss Ucebn. Zaved. Mat. 2 (1958), 118-135 (in Russian). 